\title{
Sphingolipid Metabolism and Transport in Chlamydia trachomatis and Chlamydia psittaci Infections
}

\author{
Sebastian Banhart, Elena K. Schäfer, Jean-Marc Gensch and Dagmar Heuer* \\ Unit 'Sexually Transmitted Bacterial Infections', Department for Infectious Diseases, Robert Koch Institute, Berlin, Germany
}

Chlamydia species infect a large range of vertebral hosts and have become of major economic and public health concern over the last decades. They are obligate intracellular bacteria that undergo a unique cycle of development characterized by the presence of two distinct bacterial forms. After infection of the host cell, Chlamydia are found inside a membrane-bound compartment, the inclusion. The surrounding membrane of the inclusion contributes to the host-Chlamydia interface and specific

OPEN ACCESS

Edited by:

Sibylle Schneider-Schaulies,

Julius Maximilian University of Würzburg, Germany

Reviewed by:

Vera Kozjak-Pavlovic, Julius Maximilian University of Würzburg, Germany Maria Teresa Damiani, Instituto de Histología y Embriología

Dr. Mario H. Burgos (IHEM CONICET), Argentina

*Correspondence: Dagmar Heuer heuerd@rki.de

Specialty section: This article was submitted to Membrane Physiology and Membrane Biophysics,

a section of the journal Frontiers in Cell and Developmental

Biology

Received: 28 June 2019 Accepted: 20 September 2019

Published: 04 October 2019

Citation:

Banhart S, Schäfer EK, Gensch J-M and Heuer D (2019)

Sphingolipid Metabolism

and Transport in Chlamydia trachomatis and Chlamydia psittaci Infections.

Front. Cell Dev. Biol. 7:223 doi: 10.3389/fcell.2019.00223 pathogen-derived Inc proteins shape this interface allowing interactions with distinct cellular proteins. In contrast to many other bacteria, Chlamydia species acquire sphingomyelin from the host cell. In recent years a clearer picture of how Chlamydia trachomatis acquires this lipid emerged showing that the bacteria interact with vesicular and non-vesicular transport pathways that involve the recruitment of specific RAB proteins and the lipid-transfer protein CERT. These interactions contribute to the development of a new sphingomyelin-producing compartment inside the host cell. Interestingly, recruitment of CERT is conserved among different Chlamydia species including Chlamydia psittaci. Here we discuss our current understanding on the molecular mechanisms used by $C$. trachomatis and $C$. psittaci to establish these interactions and to create a novel sphingomyelin-producing compartment inside the host cell important for the infection.

Keywords: Chlamydia, sphingolipid, sphingomyelin (SM), ceramide (CER), CERT (CERamide Transfer protein), Inc proteins, infection, RAB proteins

\section{INTRODUCTION}

Lipids are important factors in bacterial infections. They serve as energy source, structural components and are involved in the immune response. Like many bacteria, Chlamydia trachomatis is able to synthesize most phospholipids except for sphingomyelin, cholesterol and phosphatidylcholine. Sphingomyelin is mainly produced by eukaryotic cells thus; the detection of sphingomyelin inside chlamydial cells was astonishing. This review summarizes recent advances in our understanding of how Chlamydia spp. acquire sphingolipids from the host cell and describes their functions for Chlamydia biology.

\section{CLINICS OF Chlamydia trachomatis AND C. psittaci INFECTIONS}

Chlamydia trachomatis strains can be divided into biovars. The trachoma biovar (serovars A-C) can cause trachoma, the leading cause of preventable blindness that is hyperendemic in many rural 
areas of Africa, Central and South America, Asia, Australia and the Middle East. Infections with the urogenital tract biovar (serovars D-K) are among the most frequently sexually transmitted bacterial infections world-wide. They affect mainly young adults and persons with multiple sex partners (Newman et al., 2015). Symptoms range from asymptomatic to urethritis and proctitis in both genders and cervicitis in females. In particular, untreated or re-occurring infections in women have been associated with severe outcomes including pelvic inflammatory diseases (PID), ectopic pregnancies and infertility. Furthermore, during pregnancy, untreated C. trachomatis infections are a risk factor of preterm birth, conjunctivitis and pneumonia of the newborn. C. trachomatis belonging to the lymphogranuloma venereum biovar (LGV, L1-L3) is also sexually transmitted and can cause urogenital or anorectal infections in humans that can be more invasive by disseminating to the lymph nodes (Elwell et al., 2016).

Chlamydia psittaci is a zoonotic pathogen that causes respiratory disease in humans and avian species, also known as psittacosis or ornithosis (Knittler et al., 2014; Knittler and Sachse, 2015). The agent was originally isolated from birds, but meanwhile it has been found in different mammalian hosts like cattle, horses and pigs (Longbottom and Coulter, 2003). C. psittaci can be transmitted from domestic birds to humans by inhalation of aerosolized bacteria from the feces of infected avian species (Knittler et al., 2014; Knittler and Sachse, 2015). In many cases $C$. psittaci infections remain undetected and undiagnosed due to unspecific symptoms (fever, chills, headache, malaise, myalgia) (Knittler et al., 2014; Knittler and Sachse, 2015).

\section{BIOLOGY OF Chlamydiaceae}

Both C. trachomatis and C. psittaci belong to the family of Chlamydiaceae. A hallmark of all members of this family is their obligate intracellular, biphasic cycle of development that takes place in a membrane-bound compartment inside a eukaryotic host cell (Moulder, 1991; Hybiske, 2015).

It is characterized by the switch between the extracellular, infectious elementary bodies (EBs) and the intracellular, non-infectious, metabolically active reticulate bodies (RBs) (Figure 1). EBs are $0.3 \mu \mathrm{m}$ in size and enter the host cell by receptor-mediated endocytosis or phagocytosis, involving bacterial adhesins, host cell receptors, and host-specific heparan proteoglycans (Elwell et al., 2016). After internalization, EBs are found in vacuoles, termed inclusions that protect the bacteria from the immune response of the host cell. By releasing effector molecules into the host cell via a type III secretion system, the inclusion membrane is modified and can escape the phagolysosomal pathway (Moore and Ouellette, 2014). Within the inclusion, EBs differentiate into the osmotically instable RBs. These $1 \mu \mathrm{m}$ small, structurally flexible bacteria divide asymmetrically (Nunes and Gomes, 2014; Abdelrahman et al., 2016). RBs synthesize a family of special proteins, the Inc proteins, which are unique to Chlamydia spp. and are integral parts of the bacterial inclusion membrane. They are important bacterial constituents of the inclusion-host cell interface and confer stability to the inclusion membrane (Mirrashidi et al., 2015; Weber et al., 2017). Inc proteins were originally identified in C. psittaci. They are a family of Chlamydia-specific proteins lacking sequence homology to any known proteins, or to themselves. Interestingly, genomic comparison of different Chlamydia strains showed that some Inc proteins are conserved between different species and others are species-specific. These non-conserved Inc proteins may be involved in tissue tropism (Dehoux et al., 2011; Lutter et al., 2012).

At 16-20 h post infection (p.i.) some RBs start to transform back into EBs, while other RBs continue to replicate. Depending on chlamydial species and growth conditions, at 48-72 h p.i. both developmental stages are released from the host cell by either complete lysis of the host cell or by a mechanism called extrusion - the release of the intact inclusion enveloped by host cell plasma membrane (Figure 1; Hybiske and Stephens, 2007). Freed EBs can infect neighboring host cells and start a new round of infection.

\section{SPHINGOLIPID SYNTHESIS IN EUKARYOTIC CELLS}

Sphingolipids are major integral components of eukaryotic cell membranes. They function as structural and signaling molecules that can regulate apoptosis, cellular proliferation and stress responses (Heung et al., 2006; Breslow and Weissman, 2010). Defects in sphingolipid metabolism have been linked to different diseases including carcinogenesis, cardiovascular and neurodegenerative diseases (Heung et al., 2006).

A sphingoid base linked to a specific fatty acid is the building block of the diverse family of sphingolipids. In sphingomyelin, this backbone is linked to a head group of phosphocholine whereas complex glycosphingolipids are generated by addition of a specific sugar residue. Sphingolipids are a family of structurally and functionally diverse lipids and are synthesized by three distinct pathways: (1) de novo synthesis, (2) sphingomyelinase pathways, and (3) salvage pathway that involve specific enzymes localized to distinct organelles inside the cell.

De novo sphingolipid synthesis begins with condensation of serine and palmitoyl coenzyme A (CoA) which takes place at the cytosolic leaflet of the endoplasmic reticulum (ER) catalyzed by the highly conserved palmitoyltransferase (SPTLC) (Yard et al., 2007; Breslow and Weissman, 2010; Hannun and Obeid, 2018). The product of SPTLC, 3ketosphinganine, is further reduced by 3-ketosphinganine reductase (KDSR) and $\mathrm{N}$-acylated by the action of fatty acid specific dihydroceramide synthases (CERS1-6). Finally, dihydroceramide is desaturated by dihydroceramide desaturase (DEGS) to generate ceramide. Ceramide represents the central precursor molecule of the sphingolipid metabolism, which in turn is used to generate several sphingolipids, like sphingomyelin, sphingosine or complex glycosphingolipids. Ceramide is then transported from the ER to the Golgi apparatus by vesicular trafficking or by transport proteins (Bartke and Hannun, 2009; Hanada, 2010). Within the Golgi, ceramides are modified at the head-group position by adding phosphocholine and phosphate 


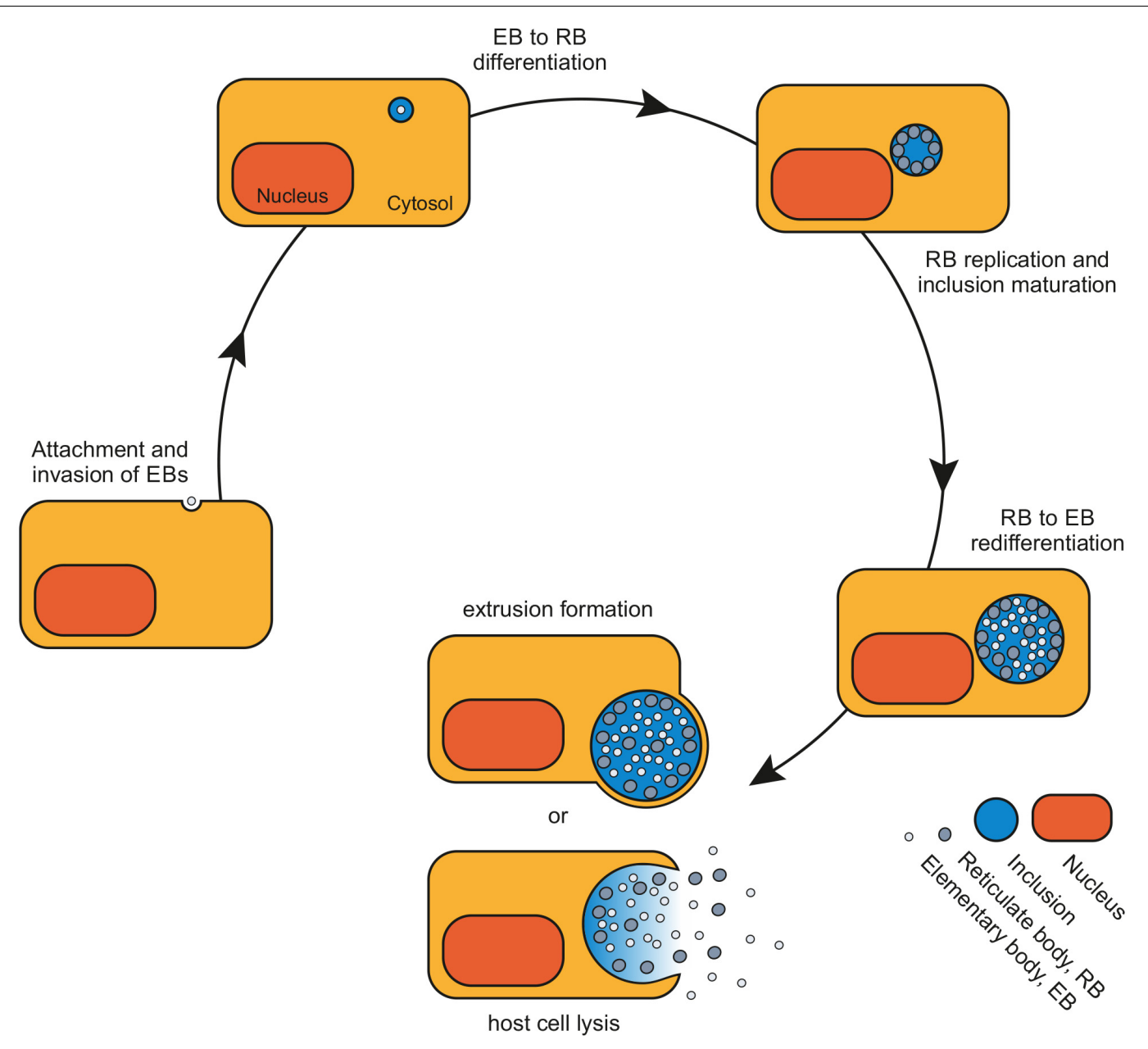

FIGURE 1 | The chlamydial developmental cycle. The biphasic developmental cycle of Chlamydia spp. starts with the attachment and invasion of host cells by infectious elementary bodies (EBs). Within their membrane-bound vacuole, termed inclusion, EBs differentiate into metabolically active reticulate bodies (RBs). RBs undergo repeated cycles of replication before they finally re-differentiate into EBs. The life cycle ends with the release of EBs from the host cell by either host cell lysis or extrusion formation to start a new round of infection.

to produce sphingomyelin and ceramide 1-phosphate (Tafesse et al., 2006; Hannun and Obeid, 2018). Ceramide is converted to sphingomyelin by sphingomyelin synthases (SMS) located at the lumen of the trans-Golgi (SMS1 and SMS2) and at the plasma membrane (SMS2) (Tafesse et al., 2006; Yamaji and Hanada, 2015). Precursor of complex glycosphingolipids, such as glucosylceramide and galactosylceramide, are formed by the addition of glucose and galactose residues in a glycosidic linkage to ceramide (Breslow and Weissman, 2010; Yamaji and Hanada, 2015). Ultimately, sphingolipids and glycosphingolipids are transported through secretory pathways to plasma membranes and subcellular organelles.

Alternatively, ceramides can be generated by the breakdown of complex sphingolipids, termed salvage pathway (Kitatani et al., 2008). Sphingolipids and glycosphingolipids are degraded in acidic subcellular compartments, such as late endosomes and lysosomes, to form sphingosine (Kitatani et al., 2008). In contrast to ceramide, which is not capable to leave the lysosome, sphingosine is able to enter different cell compartments
(Bartke and Hannun, 2009). Released sphingosine may re-enter sphingolipid pathways and is reused by the ceramide synthase to generate ceramides again via re-acylation (Kitatani et al., 2008).

The third pathway, termed sphingomyelinase pathway, occurs in the plasma membrane and endosome/lysosome systems (Yamaji and Hanada, 2015; Teo et al., 2016). Within these compartments, sphingomyelin is converted to ceramide by acid sphingomyelinases (Kitatani et al., 2008). At plasma membranes, SMS2 adds phosphocholine head groups to ceramide, which leads to the production of sphingomyelin.

\section{SPHINGOLIPID TRANSPORT IN Chlamydia-INFECTED CELLS}

Twenty four years ago, Hackstadt et al. (1995) showed that fluorescently labeled sphingomyelin is acquired by C. trachomatis from the host cell. Based on the observation that purified EBs contained fluorescent sphingomyelin the authors concluded 
that Golgi-derived sphingomyelin accumulates in bacteria rather than its precursor ceramide. Classical protein markers of the transport between the Golgi apparatus and the plasma membrane were not found in the inclusion membrane suggesting that a subset of Golgi-derived exocytic vesicles is targeted (Hackstadt et al., 1996; Scidmore et al., 1996a). C. trachomatis protein synthesis is required for this interaction and bacterial factors that mediate fusogenicity with sphingomyelin-containing vesicles seem to be continually replenished (Scidmore et al., 1996b, 2003). Shortly after these initial observations, quantitative analysis indicated that $C$. trachomatis membranes contain up to $4 \%$ of sphingolipids (Wylie et al., 1997). Interfering with bacterial sphingolipid acquisition resulted in less infectious bacteria, leads to the formation of aberrant chlamydial forms and demonstrated the requirement of sphingolipid metabolism for reactivation after INF $\gamma$ treatment of $C$. trachomatis-infected cells (van Ooij et al., 2000; Rejman Lipinski et al., 2009; Robertson et al., 2009). Interestingly, synthesis of sphingomyelin from ceramide seems to be a prerequisite for sphingolipid uptake into the inclusion and into the bacteria, as a ceramide derivative that cannot be converted to sphingomyelin (1-Omethyl-ceramide) was not translocated across the inclusion membrane but rather accumulated around the inclusion (Banhart et al., 2014). This ceramide derivative showed strong antichlamydial activity suggesting that $C$. trachomatis generates a sphingomyelin-producing compartment inside the host cell which is important for chlamydial growth (Banhart et al., 2014; Saied et al., 2015). Surprisingly, a recent study suggests that sphingomyelin uptake by Chlamydia species is linked to host adaptation and/or virulence rather than to its obligate intracellular life style (Dille et al., 2015).

In recent years, vesicular and non-vesicular transport pathways were identified that were hijacked by C. trachomatis to obtain sphingolipids from the host cell (Figure 2). These pathways are not redundant and play distinct roles during the chlamydial cycle of development. It has been shown that C. trachomatis can intercept vesicular transport routes from different organelles including Golgi mini-stacks or multivesicular bodies (MVBs). Transport of sphingolipid-containing vesicles derived from Golgi mini-stacks requires cellular GTPases RAB14, RAB6A and RAB11A, ARF1 and its guanine nucleotide exchange factor GBF1 (Heuer et al., 2009; Elwell et al., 2011). Interestingly, RAB14, RAB6A, and RAB11A appear to be important for Chlamydia progeny formation whereas ARF1 and GBF1 seem
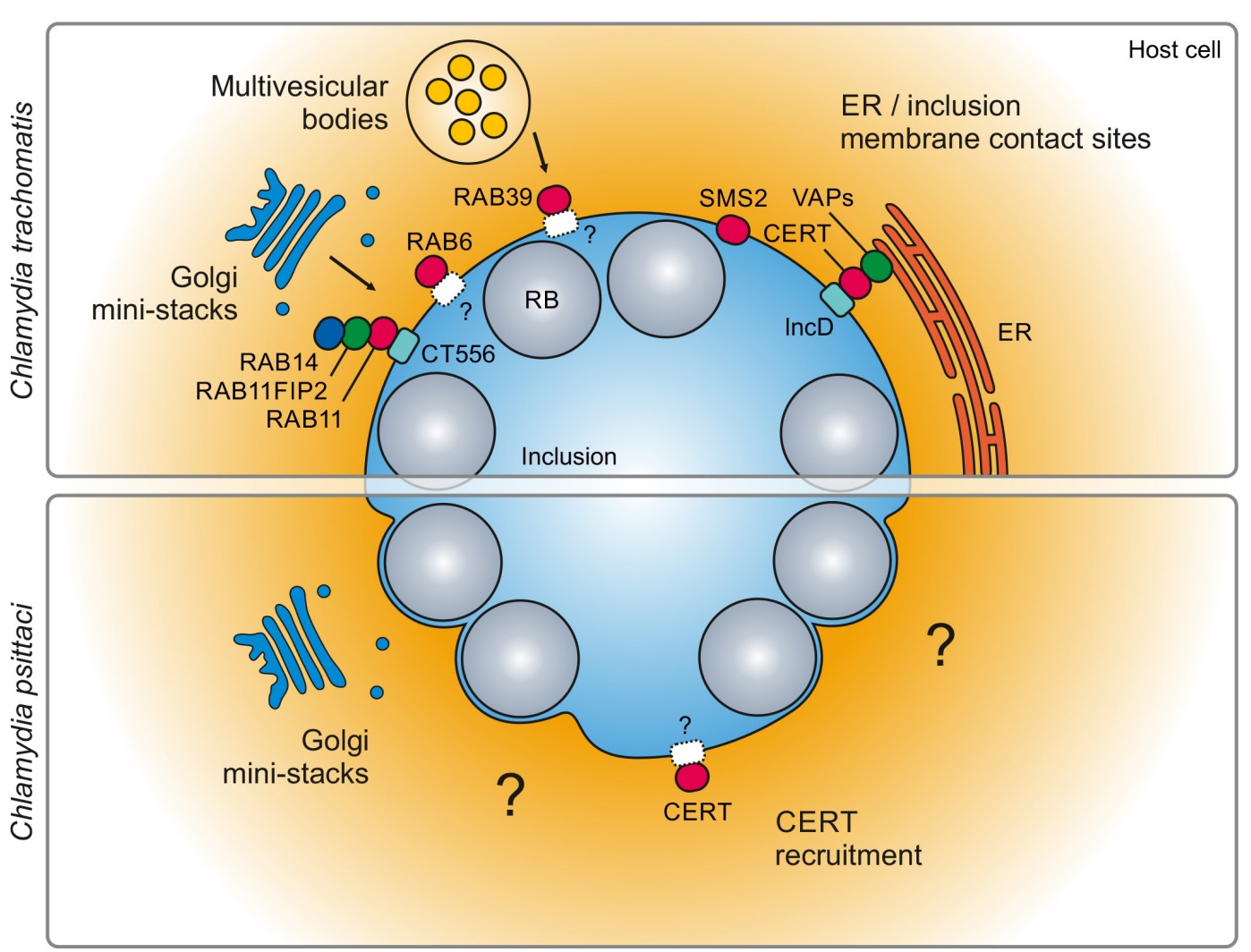

FIGURE 2 | Sphingolipid acquisition during Chlamydia infection. Acquisition of sphingolipids takes place by both vesicular and non-vesicular pathways and is ensured by interactions with several subcellular compartments and host cell proteins. Vesicular transport of sphingolipids to $C$. trachomatis is realized by rerouting vesicles from fragmented Golgi mini-stacks or multivesicular bodies, involving several RAB GTPases such as RAB6, RAB11, RAB14, and RAB39. Recruitment of RAB GTPases is thought to be mediated by Inc proteins and interaction of RAB11 with CT556 has been described (Mirrashidi et al., 2015). Non-vesicular routes to C. trachomatis include recruitment of SMS and the formation of ER/inclusion membrane contact sites that contain the ceramide transport protein CERT. In contrast, little is known for C. psittaci, except for fragmentation of the Golgi apparatus and recruitment of CERT. 
to be dispensable (Rejman Lipinski et al., 2009; Capmany and Damiani, 2010; Elwell et al., 2011). The recruitment of RAB14-postive vesicles was shown to be controlled by the Akt signaling pathway, a pathway that is activated by C. trachomatis infections (Capmany et al., 2019). Several other kinases have also been implicated in sphingomyelin transport to the C. trachomatis inclusion. These include SRC family kinase Fyn and serine/threonine kinases that have been identified in an RNAi screen and based upon inhibition by rotterlin, respectively (Shivshankar et al., 2008; Mital and Hackstadt, 2011). The precise mechanisms how rotterlin inhibits sphingomyelin uptake by C. trachomatis remains elusive as rotterlin appears to have multiple targets inside the host cell (Lei et al., 2012). In addition, sphingomyelin is transported from MVBs by RAB39 (Gambarte Tudela et al., 2019). The MVB marker protein CD63 has been detected inside C. trachomatis inclusion but its functional role remains elusive (Beatty, 2006, 2008). In contrast, much less is known for sphingolipid acquisition in C. psittaci infections (Figure 2). For both species, infection results in fragmentation of the cellular Golgi apparatus into smaller Golgi mini-stacks thereby increasing Golgi surface (Heuer et al., 2009; Knittler et al., 2014). This phenotype has been shown to boost sphingolipid acquisition in C. trachomatis infections (Heuer et al., 2009). In sum, multiple cellular processes contribute to sphingolipid acquisition in Chlamydia infections (Moore, 2012). How these factors regulate sphingolipid transport and influence the infection is currently not completely understood.

The recruitment of cellular proteins, especially RAB proteins, is species dependent (Damiani et al., 2014). In the past, the localizations of $\mathrm{RAB}$ proteins were investigated during infection of different Chlamydia species and showed that a core subset of $\mathrm{RAB}$ proteins is recruited to the inclusion membrane of different Chlamydia species whereas a few RAB proteins are species-specific (Rzomp et al., 2003). Interestingly, although recruitment of a $\mathrm{RAB}$ protein is conserved between different Chlamydia species (Rab4 in C. trachomatis serovar L2 and D, C. muridarum, and C. pneumoniae), its identified bacterial interaction partner that is responsible for the interaction (CT229 in C. trachomatis serovar L2) has not been found in the other chlamydial species (Rzomp et al., 2006). This leaves a question mark on of how the mechanisms of functional recruitment differ between Chlamydia species.

Future research regarding the role of these different vesicular pathways in infections with different Chlamydia species, the identification of transported lipids and bacterial factors controlling these interactions is needed to understand the intricate relationship.

\section{CERT-DEPENDENT ACQUISITION OF SPHINGOLIPIDS AND BEYOND}

New studies showed that C. trachomatis and C. psittaci hijack the cellular ceramide transport protein CERT to obtain ceramide from the host cell (Derre et al., 2011; Koch-Edelmann et al., 2017). CERT transfers ceramide from the ER to the Golgi apparatus in uninfected cells using the C-terminal START domain (Ponting and Aravind, 1999). Its N-terminal pleckstrin homology (PH) domain binds phosphatidylinositol-4-phosphate (PI4P) (Peretti et al., 2008) at the cis-face of the Golgi apparatus and is linked with the ER due to its central FFAT motif binding VAPs (Vesicleassociated membrane protein-associated protein) (Loewen et al., 2003). In C. trachomatis-infected cells, CERT is recruited to the inclusion membrane by interaction with $\mathrm{IncD}$ (Figure 2). Targeted deletion of CERT domains showed that the FFAT motif is relevant for binding and co-recruiting VAPs to the inclusion membrane, but lack of the $\mathrm{PH}$ domain interrupts association to the inclusion (Agaisse and Derre, 2014). The interaction of IncD with CERT is driven by the charged and hydrophobic motif in its C-terminus as well as the charged motif in the N-terminus (Kumagai et al., 2018). These motifs are conserved in C. trachomatis, C. suis, C. muridarum, C. caviae, and C. felis. Also, the proximity of both domains and the possibility of forming homooligomers mediated by the transmembrane domain are necessary for increasing the affinity to CERT. After CERT recruitment to the inclusion membrane, ceramide is likely transported from the ER to the inclusion membrane at ERinclusion contact sides where ceramide is subsequently converted into sphingomyelin by the also recruited host SMS2 (Figure 2; Elwell et al., 2011). IncD belongs to the non-conserved Inc proteins that are not found in all Chlamydia species, for example C. psittaci. Thus, it is currently not known how CERT is recruited to $C$. psittaci inclusions (Koch-Edelmann et al., 2017). In uninfected cells, the CERT PH domain binds to PI4P-enriched membranes in the trans-Golgi region. It has been suggested, that PI4P is present at C. trachomatis inclusion membranes and might thereby partially mediate CERT binding (Moorhead et al., 2010). Assuming that C. psittaci inclusions are PI4P positive, this mode of binding could be conserved between the different Chlamydia species. In addition, proteomic analysis of C. trachomatis inclusions revealed that VAPB, a binding partner of CERT is significantly enriched in the inclusion proteome (Aeberhard et al., 2015). Whether VAPB is also associated with C. psittaci inclusions or if a currently unknown C. psittaci factor facilitates CERT recruitment still needs to be determined. Thus, future experiments are needed to reveal the nature of CERT binding to C. psittaci inclusions.

Sphingomyelin is one of the essential host-derived lipids that is incorporated into chlamydial membranes (Saka and Valdivia, 2010) and is described to play a role in bacterial replication and inclusion growth (Hackstadt et al., 1996; Rejman Lipinski et al., 2009; Elwell et al., 2011). Further evidence for this suggestion is that CERT recruitment is conserved among Chlamydia spp. (Koch-Edelmann et al., 2017). For C. trachomatis and C. muridarum it has been shown by RNA interference that CERT seems to be essential for the production of infectious progeny, indicating that CERT is a crucial factor in chlamydial development (Derre et al., 2011; Elwell et al., 2011). Recent studies using CRISPR/Cas9-mediated CERT-knockout cells demonstrated that deficiency of CERT in C. psittaci infections also leads to decreased infectious progeny formation (Koch-Edelmann et al., 2017). Interestingly, CERT-knockout caused an increase of sphingolipid uptake by C. psittaci (KochEdelmann et al., 2017). This is in stark contrast to C. trachomatis 
infection that shows a drastic decrease in bacterial sphingolipid acquisition under CERT depletion. These findings possibly suggest a CERT-independent sphingolipid uptake pathway in C. psittaci infections. How sphingolipids are transported to C. psittaci in CERT-knockout cells is currently not known. The involvement of one or more novel factor/s of either bacterial and/or cellular origin that compensate for loss of CERT is likely. Besides that, these results underline that acquisition of sphingomyelin needs to be controlled by Chlamydia spp. and suggest that CERT might have additional roles in chlamydial development beyond sphingolipid transport, which need to be investigated in the future.

\section{SUMMARY AND OUTLOOK}

Twenty four years after the initial observation that C. trachomatis can acquire sphingomyelin from the Golgi apparatus of the infected host cells a clearer picture is emerging on the molecular pathways used by different Chlamydia species to obtain sphingolipids. Chlamydia species use distinct, non-redundant pathways to obtain sphingolipids. These include vesicular and non-vesicular transport pathways. The characterization of CERT as a conserved factor in ceramide delivery to different Chlamydia species and the recruitment of the human SMS2 to the C. trachomatis inclusion suggests that at least C. trachomatis creates a novel sphingomyelin-producing compartment inside the infected host cells. Additionally, in C. trachomatis infections sphingomyelin is transported by distinct vesicles. For that purpose, C. trachomatis exploits cellular GTPases, including

\section{REFERENCES}

Abdelrahman, Y., Ouellette, S. P., Belland, R. J., and Cox, J. V. (2016). Polarized cell division of Chlamydia trachomatis. PLoS Pathog. 12:e1005822. doi: 10.1371/ journal.ppat.1005822

Aeberhard, L., Banhart, S., Fischer, M., Jehmlich, N., Rose, L., Koch, S., et al. (2015). The proteome of the isolated Chlamydia trachomatis containing vacuole reveals a complex trafficking platform enriched for retromer components. PLoS Pathog. 11:e1004883. doi: 10.1371/journal.ppat.1004883

Agaisse, H., and Derre, I. (2014). Expression of the effector protein IncD in Chlamydia trachomatis mediates recruitment of the lipid transfer protein CERT and the endoplasmic reticulum-resident protein VAPB to the inclusion membrane. Infect. Immun. 82, 2037-2047. doi: 10.1128/IAI.01530- 14

Banhart, S., Saied, E. M., Martini, A., Koch, S., Aeberhard, L., Madela, K., et al. (2014). Improved plaque assay identifies a novel anti-Chlamydia ceramide derivative with altered intracellular localization. Antimicrob. Agents Chemother. 58, 5537-5546. doi: 10.1128/AAC.03457-14

Bartke, N., and Hannun, Y. A. (2009). Bioactive sphingolipids: metabolism and function. J. Lipid Res. 50(Suppl.), S91-S96.

Beatty, W. L. (2006). Trafficking from CD63-positive late endocytic multivesicular bodies is essential for intracellular development of Chlamydia trachomatis. J. Cell Sci. 119, 350-359. doi: 10.1242/jcs.02733

Beatty, W. L. (2008). Late endocytic multivesicular bodies intersect the chlamydial inclusion in the absence of CD63. Infect. Immun. 76, 2872-2881. doi: 10.1128/ IAI.00129-08

Breslow, D. K., and Weissman, J. S. (2010). Membranes in balance: mechanisms of sphingolipid homeostasis. Mol. Cell 40, 267-279. doi: 10.1016/j.molcel.2010. 10.005

Capmany, A., and Damiani, M. T. (2010). Chlamydia trachomatis intercepts Golgi-derived sphingolipids through a Rab14-mediated transport required
$\mathrm{RAB}$ and $\mathrm{ARF}$ proteins, and kinases to facilitate bacterial sphingomyelin acquisition from fragmented Golgi mini-stacks and MVBs. How vesicular and non-vesicular transport of sphingolipids is controlled by different Chlamydia species, how they process CERT-delivered ceramide, and how ceramide and sphingomyelin regulate chlamydial infections are just a few open questions. The development of novel tools including the genetic manipulation of Chlamydia species and the biochemical isolation of chlamydial inclusions now allows addressing these questions.

\section{AUTHOR CONTRIBUTIONS}

$\mathrm{DH}$ developed the conception of the manuscript and wrote the manuscript. SB designed the figures. ES, J-MG, and SB contributed to and wrote sections of the manuscript. All authors contributed to the manuscript revision, read, and approved the submitted version of the manuscript.

\section{FUNDING}

This work was supported by the Deutsche Forschungsgemeinschaft (SPP 1580 to DH, HE6008/1-1 and HE6008/1-2) and the Federal Ministry of Education and Research (BMBF) in the framework of the national research network "Zoonotic Chlamydia - Models of chronic and persistent infections in humans and animals." We apologize to those investigators whose work we were unable to cite because of space constraints.

for bacterial development and replication. PLoS One 5:e14084. doi: 10.1371/ journal.pone.0014084

Capmany, A., Gambarte Tudela, J., Alonso Bivou, M., and Damiani, M. T. (2019). Akt/AS160 signaling pathway inhibition impairs infection by decreasing Rab14-controlled sphingolipids delivery to Chlamydial Inclusions. Front. Microbiol. 10:666. doi: 10.3389/fmicb.2019.00666

Damiani, M. T., Gambarte Tudela, J., and Capmany, A. (2014). Targeting eukaryotic Rab proteins: a smart strategy for chlamydial survival and replication. Cell. Microbiol. 16, 1329-1338. doi: 10.1111/cmi.12325

Dehoux, P., Flores, R., Dauga, C., Zhong, G., and Subtil, A. (2011). Multi-genome identification and characterization of chlamydiae-specific type III secretion substrates: the Inc proteins. BMC Genomics 12:109. doi: 10.1186/1471-216412- 109

Derre, I., Swiss, R., and Agaisse, H. (2011). The lipid transfer protein CERT interacts with the Chlamydia inclusion protein IncD and participates to ER-Chlamydia inclusion membrane contact sites. PLoS Pathog. 7:e1002092. doi: 10.1371/journal.ppat.1002092

Dille, S., Kleinschnitz, E. M., Kontchou, C. W., Nolke, T., and Hacker, G. (2015). In contrast to Chlamydia trachomatis, Waddlia chondrophila grows in human cells without inhibiting apoptosis, fragmenting the Golgi apparatus, or diverting post-Golgi sphingomyelin transport. Infect. Immun. 83, 3268-3280. doi: 10.1128/IAI.00322-15

Elwell, C., Mirrashidi, K., and Engel, J. (2016). Chlamydia cell biology and pathogenesis. Nat. Rev. Microbiol. 14, 385-400. doi: 10.1038/nrmicro.2016.30

Elwell, C. A., Jiang, S., Kim, J. H., Lee, A., Wittmann, T., Hanada, K., et al. (2011). Chlamydia trachomatis co-opts GBF1 and CERT to acquire host sphingomyelin for distinct roles during intracellular development. PLoS Pathog. 7:e1002198. doi: 10.1371/journal.ppat.1002198

Gambarte Tudela, J., Buonfigli, J., Lujan, A., Alonso Bivou, M., Cebrian, I., Capmany, A., et al. (2019). Rab39a and Rab39b display different intracellular 
distribution and function in sphingolipids and phospholipids transport. Int. J. Mol. Sci. 20:E1688. doi: 10.3390/ijms20071688

Hackstadt, T., Rockey, D. D., Heinzen, R. A., and Scidmore, M. A. (1996). Chlamydia trachomatis interrupts an exocytic pathway to acquire endogenously synthesized sphingomyelin in transit from the Golgi apparatus to the plasma membrane. EMBO J. 15, 964-977. doi: 10.1002/j.1460-2075.1996.tb00433.x

Hackstadt, T., Scidmore, M. A., and Rockey, D. D. (1995). Lipid metabolism in Chlamydia trachomatis-infected cells: directed trafficking of Golgi-derived sphingolipids to the chlamydial inclusion. Proc Natl Acad Sci U.S.A. 92, 4877-4881. doi: 10.1073/pnas.92.11.4877

Hanada, K. (2010). Intracellular trafficking of ceramide by ceramide transfer protein. Proc. Jpn. Acad. Ser. B Phys. Biol. Sci. 86, 426-437. doi: 10.2183/pjab. 86.426

Hannun, Y. A., and Obeid, L. M. (2018). Sphingolipids and their metabolism in physiology and disease. Nat. Rev. Mol. Cell Biol. 19, 175-191. doi: 10.1038/nrm. 2017.107

Heuer, D., Rejman Lipinski, A., Machuy, N., Karlas, A., Wehrens, A., Siedler, F., et al. (2009). Chlamydia causes fragmentation of the Golgi compartment to ensure reproduction. Nature 457, 731-735. doi: 10.1038/nature07578

Heung, L. J., Luberto, C., and Del Poeta, M. (2006). Role of sphingolipids in microbial pathogenesis. Infect. Immun. 74, 28-39. doi: 10.1128/iai.74.1.28-39. 2006

Hybiske, K. (2015). Expanding the molecular toolkit for Chlamydia. Cell Host Microbe 18, 11-13. doi: 10.1016/j.chom.2015.06.016

Hybiske, K., and Stephens, R. S. (2007). Mechanisms of host cell exit by the intracellular bacterium Chlamydia. Proc. Natl. Acad. Sci. U.S.A. 104, 11430-11435. doi: 10.1073/pnas.0703218104

Kitatani, K., Idkowiak-Baldys, J., and Hannun, Y. A. (2008). The sphingolipid salvage pathway in ceramide metabolism and signaling. Cell. Signal. 20, 1010-1018. doi: 10.1016/j.cellsig.2007.12.006

Knittler, M. R., Berndt, A., Bocker, S., Dutow, P., Hanel, F., Heuer, D., et al. (2014). Chlamydia psittaci: new insights into genomic diversity, clinical pathology, host-pathogen interaction and anti-bacterial immunity. Int. J. Med. Microbiol. 304, 877-893. doi: 10.1016/j.ijmm.2014.06.010

Knittler, M. R., and Sachse, K. (2015). Chlamydia psittaci: update on an underestimated zoonotic agent. Pathog. Dis. 73, 1-15. doi: 10.1093/femspd/ $\mathrm{ftu} 007$

Koch-Edelmann, S., Banhart, S., Saied, E. M., Rose, L., Aeberhard, L., Laue, M., et al. (2017). The cellular ceramide transport protein CERT promotes Chlamydia psittaci infection and controls bacterial sphingolipid uptake. Cell. Microbiol. 19:e12752. doi: 10.1111/cmi.12752

Kumagai, K., Elwell, C. A., Ando, S., Engel, J. N., and Hanada, K. (2018). Both the $\mathrm{N}$ - and C-terminal regions of the Chlamydial inclusion protein D ( IncD) are required for interaction with the pleckstrin homology domain of the ceramide transport protein CERT. Biochem. Biophys. Res. Commun. 505, 1070-1076. doi: 10.1016/j.bbrc.2018.09.168

Lei, L., Li, Z., and Zhong, G. (2012). Rottlerin-mediated inhibition of Chlamydia trachomatis growth and uptake of sphingolipids is independent of $\mathrm{p} 38$ regulated/activated protein kinase (PRAK). PLoS One 7:e44733. doi: 10.1371/ journal.pone. 0044733

Loewen, C. J., Roy, A., and Levine, T. P. (2003). A conserved ER targeting motif in three families of lipid binding proteins and in Opilp binds VAP. EMBO J. 22, 2025-2035. doi: 10.1093/emboj/cdg201

Longbottom, D., and Coulter, L. J. (2003). Animal chlamydioses and zoonotic implications. J. Comp. Pathol. 128, 217-244. doi: 10.1053/jcpa.2002.0629

Lutter, E. I., Martens, C., and Hackstadt, T. (2012). Evolution and conservation of predicted inclusion membrane proteins in chlamydiae. Comp. Funct. Genomics 2012:362104. doi: 10.1155/2012/362104

Mirrashidi, K. M., Elwell, C. A., Verschueren, E., Johnson, J. R., Frando, A., Von Dollen, J., et al. (2015). Global mapping of the Inc-human interactome reveals that retromer restricts chlamydia infection. Cell Host Microbe 18, 109-121. doi: 10.1016/j.chom.2015.06.004

Mital, J., and Hackstadt, T. (2011). Role for the SRC family kinase Fyn in sphingolipid acquisition by chlamydiae. Infect. Immun. 79, 4559-4568. doi: 10.1128/IAI.05692-11

Moore, E. R. (2012). Sphingolipid trafficking and purification in Chlamydia trachomatis-infected cells. Curr. Protoc. Microbiol. Chapter 11:2.
Moore, E. R., and Ouellette, S. P. (2014). Reconceptualizing the chlamydial inclusion as a pathogen-specified parasitic organelle: an expanded role for Inc proteins. Front. Cell. Infect. Microbiol. 4:157. doi: 10.3389/fcimb.2014. 00157

Moorhead, A. M., Jung, J. Y., Smirnov, A., Kaufer, S., and Scidmore, M. A. (2010). Multiple host proteins that function in phosphatidylinositol-4-phosphate metabolism are recruited to the chlamydial inclusion. Infect. Immun. 78, 1990-2007. doi: 10.1128/IAI.01340-09

Moulder, J. W. (1991). Interaction of chlamydiae and host cells in vitro. Microbiol. Rev. 55, 143-190.

Newman, L., Rowley, J., Vander Hoorn, S., Wijesooriya, N. S., Unemo, M., Low, N., et al. (2015). Global estimates of the prevalence and incidence of four curable sexually transmitted infections in 2012 based on systematic review and global reporting. PLoS One 10:e0143304. doi: 10.1371/journal.pone.0143304

Nunes, A., and Gomes, J. P. (2014). Evolution, phylogeny, and molecular epidemiology of Chlamydia. Infect. Genet. Evol. 23, 49-64. doi: 10.1016/j. meegid.2014.01.029

Peretti, D., Dahan, N., Shimoni, E., Hirschberg, K., and Lev, S. (2008). Coordinated lipid transfer between the endoplasmic reticulum and the Golgi complex requires the VAP proteins and is essential for Golgi-mediated transport. Mol. Biol. Cell 19, 3871-3884. doi: 10.1091/mbc.E08-05-0498

Ponting, C. P., and Aravind, L. (1999). START: a lipid-binding domain in StAR, HD-ZIP and signalling proteins. Trends Biochem. Sci. 24, 130-132. doi: 10.1016/s0968-0004(99)01362-6

Rejman Lipinski, A., Heymann, J., Meissner, C., Karlas, A., Brinkmann, V., Meyer, T. F., et al. (2009). Rab6 and Rab11 regulate Chlamydia trachomatis development and golgin-84-dependent Golgi fragmentation. PLoS Pathog. 5:e1000615. doi: 10.1371/journal.ppat.1000615

Robertson, D. K., Gu, L., Rowe, R. K., and Beatty, W. L. (2009). Inclusion biogenesis and reactivation of persistent Chlamydia trachomatis requires host cell sphingolipid biosynthesis. PLoS Pathog. 5:e1000664. doi: 10.1371/journal. ppat.1000664

Rzomp, K. A., Moorhead, A. R., and Scidmore, M. A. (2006). The GTPase Rab4 interacts with Chlamydia trachomatis inclusion membrane protein CT229. Infect. Immun. 74, 5362-5373. doi: 10.1128/iai.00539-06

Rzomp, K. A., Scholtes, L. D., Briggs, B. J., Whittaker, G. R., and Scidmore, M. A. (2003). Rab GTPases are recruited to chlamydial inclusions in both a speciesdependent and species-independent manner. Infect. Immun. 71, 5855-5870. doi: $10.1128 /$ iai.71.10.5855-5870.2003

Saied, E. M., Banhart, S., Burkle, S. E., Heuer, D., and Arenz, C. (2015). A series of ceramide analogs modified at the 1-position with potent activity against the intracellular growth of Chlamydia trachomatis. Future Med. Chem. 7, 1971-1980. doi: 10.4155/fmc.15.126

Saka, H. A., and Valdivia, R. H. (2010). Acquisition of nutrients by Chlamydiae: unique challenges of living in an intracellular compartment. Curr. Opin. Microbiol. 13, 4-10. doi: 10.1016/j.mib.2009.11.002

Scidmore, M. A., Fischer, E. R., and Hackstadt, T. (1996a). Sphingolipids and glycoproteins are differentially trafficked to the Chlamydia trachomatis inclusion. J. Cell Biol. 134, 363-374. doi: 10.1083/jcb.134.2.363

Scidmore, M. A., Rockey, D. D., Fischer, E. R., Heinzen, R. A., and Hackstadt, T. (1996b). Vesicular interactions of the Chlamydia trachomatis inclusion are determined by chlamydial early protein synthesis rather than route of entry. Infect. Immun. 64, 5366-5372.

Scidmore, M. A., Fischer, E. R., and Hackstadt, T. (2003). Restricted fusion of Chlamydia trachomatis vesicles with endocytic compartments during the initial stages of infection. Infect. Immun. 71, 973-984. doi: 10.1128/iai.71.2.973-984. 2003

Shivshankar, P., Lei, L., Wang, J., and Zhong, G. (2008). Rottlerin inhibits chlamydial intracellular growth and blocks chlamydial acquisition of sphingolipids from host cells. Appl. Environ. Microbiol. 74, 1243-1249. doi: $10.1128 /$ aem.02151-07

Tafesse, F. G., Ternes, P., and Holthuis, J. C. (2006). The multigenic sphingomyelin synthase family. J. Biol. Chem. 281, 29421-29425. doi: 10.1074/jbc.r60002 1200

Teo, W. X., Kerr, M. C., Huston, W. M., and Teasdale, R. D. (2016). Sortilin is associated with the chlamydial inclusion and is modulated during infection. Biol. Open 5, 429-435. doi: 10.1242/bio.016485 
van Ooij, C., Kalman, L., Ijzendoorn van, P., Nishijima, M., Hanada, K., Mostov, K., et al. (2000). Host cell-derived sphingolipids are required for the intracellular growth of Chlamydia trachomatis. Cell. Microbiol. 2, 627-637. doi: 10.1046/j. 1462-5822.2000.00077.x

Weber, M. M., Lam, J. L., Dooley, C. A., Noriea, N. F., Hansen, B. T., Hoyt, F. H., et al. (2017). Absence of specific Chlamydia trachomatis inclusion membrane proteins triggers premature inclusion membrane lysis and host cell death. Cell Rep. 19, 1406-1417. doi: 10.1016/j.celrep.2017.04.058

Wylie, J. L., Hatch, G. M., and McClarty, G. (1997). Host cell phospholipids are trafficked to and then modified by Chlamydia trachomatis. J. Bacteriol. 179, 7233-7242. doi: 10.1128/jb.179.23.7233-7242.1997

Yamaji, T., and Hanada, K. (2015). Sphingolipid metabolism and interorganellar transport: localization of sphingolipid enzymes and lipid transfer proteins. Traffic 16, 101-122. doi: 10.1111/tra.12239
Yard, B. A., Carter, L. G., Johnson, K. A., Overton, I. M., Dorward, M., Liu, H., et al. (2007). The structure of serine palmitoyltransferase; gateway to sphingolipid biosynthesis. J. Mol. Biol. 370, 870-886.

Conflict of Interest: The authors declare that the research was conducted in the absence of any commercial or financial relationships that could be construed as a potential conflict of interest.

Copyright (c) 2019 Banhart, Schäfer, Gensch and Heuer. This is an open-access article distributed under the terms of the Creative Commons Attribution License (CC BY). The use, distribution or reproduction in other forums is permitted, provided the original author(s) and the copyright owner(s) are credited and that the original publication in this journal is cited, in accordance with accepted academic practice. No use, distribution or reproduction is permitted which does not comply with these terms. 